\title{
Médecine basée sur les preuves et éducation médicale
}

L

es progrès biotechnologiques et les conséquences médicales économiques et éthiques qu'ils engendrent ont créé une problématique nouvelle pour la profession médicale. Le médecin est confronté à une croi ssance exponentielle des données scientifiques La divulgation de ces données est protéiforme et le praticien, quels que soi ent son niveau d'expertise et son mode d'exercice, éprouve des difficultés quasi insurmontables pour évaluer la qualité des informations, gérer les différencesvoi relescontradictionsentreles faits ou conclusions diffusées et intégrer dans la prati que les résultats des recherches. II n'est, par conséquent, pas surprenant de constater la variabilité des pratiques qui témoigne, au moins en partie, de la dimension du champ del'incertitude dans la prise de décision.

Face à cette variabilité qui interpelle les responsables de la santé publique et surtout les organismes payeurs, il est tentant de définir des référentiels qui sappuient sur des faits démontrés. Cette procédure vise à réduire les domaines de l'intuition, de la spéculation intellectuelle et de la conviction qui contribuent largement à la prise des décisions médicales. Cette médecine reposant sur les faits validés est née en Amérique du $\mathrm{N}$ ord où elle est désigné sous le terme " evidence-based medicine». Le terme " evidence» correspond à la traduction du mot « preuve ». Cette " médecine basée sur les preuves» (M BP) constitue un nouveau paradigme qui repose sur l'application dans les domaines du diagnostic, du pronostic, du traitement et de la prévention des connaissances, tenues pour certaines ou au moins admises comme telles. Ses promoteurs ont tenté, avec une certaine arrogance, d'imposer le concept comme le seul acceptable avant d'admettre, à partir de 1996 que, à côté de la M BP, il restait dans la pratique une place importante à l'intuition qui est en fait pour l'essentiel un produit dela mémoire autobiographique (ou épisodique), à l'expérience clinique et au raisonnement ${ }^{2}$. La taxonomie des niveaux de preuves, les moyens d'accéder d'une manière exhaustive et rapide aux informations publiées et la méthodologie d'élaboration des recommandations médicales et professionnelles ont été précisés.

D ans chaque pays, obéissant à des initiatives de divers organismes, des démarches similaires ont abouti à une explosion de recommandations. Le texte de Roger Ladouceur dans la rubrique Tribune $^{3}$ exprime, avec un humour parfois grinçant, les doutes et le désarroi du clinicien face à cette abondance. $D$ ans un même ordre d'idées, plusieurs publications récentes mettent en exergue les forces et les limites de la M BP et tendent à en limiter la pertinence pour la médecine au quotidien ${ }^{4-7}$. Les responsables de formation initiale ou de formation continue sont donc en droit de sinterroger sur la place que l'on doit donner à la M BP dansl'éducation médicale et sur les modalités pour apprendre à I'utiliser.

On peut trouver dans une réflexion objective, certains éléments pour tenter de répondre à ces interrogations. A ce jour, il et classique d'admettre que le champ d'application de la M BP couvre moins de $20 \%$ des situations cliniques auxquels est confronté le médecin praticien. Les " preuves » sont issues des travaux portant sur des populations sélectionnées éliminant les sujets répondant aux critères d'exclusion; elles reposent sur des données statistiques qui par leur nature comportent une part d'incertitude; enfin, elles n'intéressent que lesdomaines de cequi est explicite et quantifiable. On peut opposer au champ théorique d'application de la M BP celui de la pratique médicalequi doit gérer toutes les situations dont certaines sont complexes et mal définies, ce qui oblige souvent à prendre des décisions dans un contexte d'incertitude. Enfin, l'exercice médical sadresse à un être unique dont on doit respecter les valeurs et les pré férences. En dépit du fossé apparent entre la M BP et la médecine pratique, nul ne peut nier que la MBP fournit des outils utiles pour aider les 
praticiens à introduire les progrès scientifiques dans les démarches de soins et de santé publique. Encore faut-il en percevoir la pertinence, en accepter l'utilité et surtout savoir l'utiliser.

L'enseignement de la M BP doit prendre sa place dans l'éducation médicale comme le soulignent HG Welsh et JD Lurie ${ }^{8}$. II sagit d'un nouveau défi pour les enseignants. Trois pistes de réflexion peuvent être proposées aux enseignants :

- la M BP doit être enseignée en précisant ses forces et ses limites. $C$ et enseignement doit apprendre aux futurs médecinsà poser les bonnes questions et à accéder grâce aux nouvel les technologies de l'information aux recommandations validées;

- à côté de la M BP, il faut intensifier l'enseigne-

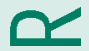
ment des aspects « non évidents » et notamment apprendre à gérer les incertitudes ;

- les enseignés doi vent être instruits du fait que la MBP trouve son champ d'application de prédilection dans les situations fréquemment

Références:

1. The Evidence-Based M edicine Working Group. Evidence-based medicine : a new approach to teaching the practice of medicine. JAMA 1992 ; $268: 2420-2425$.

2. Sackett DL, Rosenberg WM C, M uir Gray JA, Hoynes RB, R. Chardson WS. Evidence-based medicine : what it is and what it isn't. BM J $1996 ; 312: 71-72$.

3. Ladouceur R. Encore de nouvelles lignes directices ! Pédagogie M édicale ; 2 : 83 - 84.

4. $N$ aylor CD. Grey zones of clinical practice: some limits to evidence-based medicine; Lancet 1995; $345: 840$ - 842. rencontrées ou lorsque le bénéfice escompté est grand ou lorsqu'il s'agit d'un traitement à haut risque.

En ce début du XXI e siècle, le médecin doit avoir acquis une expertise pour gérer les composantes d'un nouveau paradigme, celui du « pouvoir » des malades Ceux-ci ont désormais accès aux mêmes informations que leur médecin, y compris les données de la M BP. La décision médicale sera de plus en plus une décision partagée prise dans le cadre d'un pacte de confiance. La M BP servira d'aide précieuse à la décision pour le médecin et le malade notamment dans les pathologies graves ou dans les maladies chroniques. En dépit des progrès scientifiques, on peut cependant admettre, comme l'écrivait 0 sler, que « la bonne médecine dinique devra toujours mélanger l'art de l'incertitude avec la science de la probabilité».

Raymond COLIN raymond.colin@chu-rouen.fr

5. M aynard A. Evidence-based medicine : an incomplet method for informing treatment choices. Lancet $1997 ; 349$ : 126 - 128.

6. Tonelli M R. The philosophical limits of evidencebased medicine. Academic M edicine 1998 ; $73: 1234-1240$.

7. Tanenbaum SJ. Evidence and expertise : the challenges of the outcomes movement to medical professionalism. Academic M edicine 1999 ; 74 : $757-763$.

8. Welsh HG, Lurie JD. Teaching evidence-based medicine : caveats and challenges. Academic M edicine $2000 ; 75$ : 235 - 240. 